\title{
THE INFLUENCE OF ELASTICITY ON ANALYSIS: MODERN DEVELOPMENTS
}

\author{
BY STUART S. ANTMAN
}

\author{
Contents \\ 1. Introduction \\ 2. Connectivity questions of global bifurcation theory \\ 3. The peculiarities of nonlinear elasticity \\ 4. Calculus of variations. Weak convergence \\ 5. Variational inequalities \\ 6. Other contributions \\ 7. Conclusion
}

1. Introduction. This article is one of a pair devoted to an historical study of the influence of the theory of elasticity upon the development of mathematical analysis. In the accompanying article (which appears in this issue of the Bulletin) C. Truesdell describes the contribution made by elasticity to analysis through the middle of the nineteenth century. By that time, the three-dimensional theories of linear and nonlinear elasticity had been well established. From then on, linear elasticity has enjoyed an extensive development, characterized by the use of increasingly sophisticated methods of linear analysis. Nonlinear elasticity, on the other hand, was not to receive sustained scrutiny until after the Second World War.

In this article I refrain from discussing developments in the century beginning in 1855 in order to take up the more fascinating tale of the modern interaction of nonlinear elasticity with nonlinear analysis. (St. Venant's memoir on torsion, discussed by Truesdell, appeared in 1855. Cauchy died in 1857.) Despite this gap, the subject of my account is a fitting complement to that of Truesdell, because the modern problems of nonlinear elasticity are much closer in spirit to those studied by the Bernoullis and Euler than to the linear problems studied by the successors of Cauchy. Below we examine several areas of modern analysis to which elasticity has made crucial contributions.

2. Connectivity questions of global bifurcation theory. An elastica is a mathematical model for a thin, flexible beam. Its configuration is described by a curve. Bifurcation theory began with Euler's (1744) analysis of the planar equilibrium configurations of the elastica subjected solely to end forces. This

This article represents a slightly expanded version of a lecture presented at the AMS-MAA joint meeting held in Toronto, Canada August 25, 1982; received by the editors March 15, 1983.

1980 Mathematics Subject Classification. Primary 34B15, 35J60, 35J85, 49A22, 58E07, 73C50; Secondary 35B32, 47H99, 73H05, 73Kxx. 
problem is governed by the following differential equations for the curve $[0,1] \ni s \mapsto(x(s), y(s))$ in the $(x, y)$-plane:

$$
\begin{gathered}
{\left[B(s) \theta^{\prime}(s)\right]^{\prime}+\lambda \sin \theta(s)=0,} \\
x^{\prime}(s)=\cos \theta(s), \quad y^{\prime}(s)=\sin \theta(s) .
\end{gathered}
$$

These equations are to be supplemented with a suitable set of boundary conditions. $s$ represents a scaled arc length parameter. The $x$-axis is taken parallel to the line of forces. $\theta(s)$ is the angle the tangent to the curve $(x, y)$ at $s$ makes with the $x$-axis. $\lambda$ is the magnitude of the terminal force, taken to be positive when compressive. $B(s)$, which is positive, is the stiffness of the beam at $s . B$ is not constant when the thickness is not constant.

For certain boundary conditions, such as those in which the positions and orientations of each end of the elastica are prescribed, the terminal force may have one prescribed component and one reactive component (Lagrange multiplier) that maintains the kinematical boundary condition. In this case the reactive component and hence the terminal force are not known a priori. In particular, neither $\lambda$ nor the orientation of the $x$-axis with respect to any fixed line in the plane is known a priori. When this happens the problem for $\theta$ cannot be uncoupled from that for $x$ and $y$.

For the case in which $B=$ const. Euler gave an exhaustive qualitative description of all possible solutions of (2.1), (2.2), but only a partial analysis of solutions of actual boundary value problems for these equations. (He formulated his problem entirely in terms of $x$ and $y$. His classification of all solutions of (2.1), (2.2) can be carried out more efficiently today by means of a phase-plane analysis of (2.1). The Bernoullis and Euler had, however, constructed the tools necessary to pose the governing equations in the form (2.1).) In his treatment of boundary value problems Euler studied the process of buckling, in which the trivial solution $\theta=0$, characterizing a straight configuration, loses its stability at a critical value of the parameter $\lambda$, from which there bifurcates a family of nontrivial solutions. He gave a rigorous description of the role of the linearization of (2.1), namely

$$
\left(B \psi^{\prime}\right)^{\prime}+\lambda \psi=0,
$$

in the analysis of (2.1). Fortunately Euler left some unresolved issues for his successors: He did not treat boundary value problems in which $\lambda$ was not prescribed and he did not correlate qualitative properties of solutions with the bifurcating branches on which they lay. One measure of his contribution is that to this day engineers have worked almost exclusively with linearized equations equivalent to (2.3).

It was apparently Saalschütz (1880) who first obtained closed-form solutions of boundary value problems for (2.1) with $B=$ const. in terms of Jacobi elliptic functions. (Euler had effectively determined the qualitative properties of these functions.) The use of these special functions was a mixed blessing for elasticity: Elliptic functions furnished explicit solutions for well-set nonlinear problems, but their very availability contributed to the suppression of "nonlinear thinking". (It was not until the middle of our century that workers in continuum mechanics began to treat nonlinear problems with confidence.) 
Using these explicit solutions we can efficiently display the properties of solutions of a boundary value problem for (2.1), say the problem in which

$$
\theta(0)=0=\theta^{\prime}(1) \text {, }
$$

by means of a bifurcation diagram, Figure 2.5. The ordinate labeled $\theta$ represents some convenient measure of the amplitude of the solution (e.g., $\theta^{\prime}(0)$ ). Thus the $\lambda$-axis represents the trivial solution. Any connected family of pairs $(\lambda, \theta)$ satisfying (2.1), (2.4) is a branch of solution pairs of the problem. There are a countable infinity of nontrivial solution branches bifurcating from the trivial branch at $\left(\lambda_{k}, 0\right), k=0,1,2, \ldots$, where $\lambda_{k}=(2 k+1)^{2} \pi^{2} B / 4$ is the $k$ th eigenvalue of (2.3) subject to boundary conditions of the form (2.4). Each such branch is unbounded in the space $\mathbf{R} \times C^{2}([0,1])$ of solution pairs and meets the trivial branch at only one point. On the $k$ th branch $\theta$ has exactly $k$ zeros on $(0,1)$, each of which is simple. (An account of the detailed properties of the explicit solutions of (2.1), (2.4) oriented toward bifurcation theory is given by Reiss (1969).)

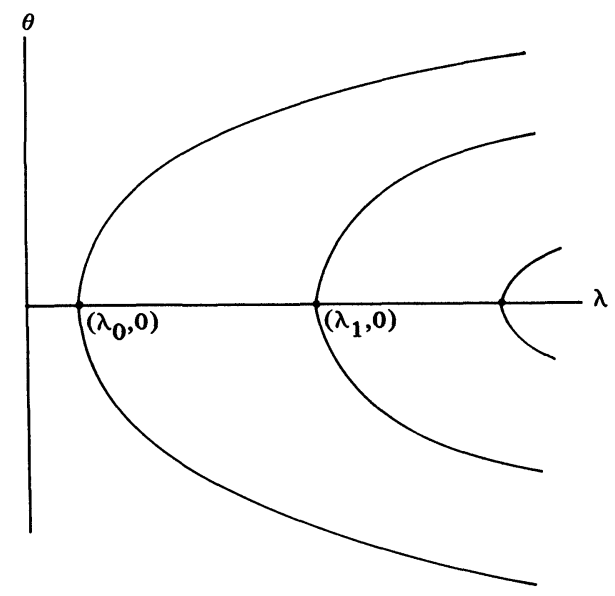

FIGURE 2.5. Bifurcation diagram for (2.1), (2.4).

Now we jump over the next seventy-five years, ignoring very important contributions to bifurcation theory by Poincaré, Lyapunov, Schmidt, Lyusternik, Shnirel'man, Krasnosel'skiri, and others in order to describe the seminal work of Kolodner (1955) on the bifurcated steady states of a rotating heavy chain. In this problem the upper end $s=1$ of the chain is held fixed and the lower end $s=0$ is left free. We seek steady states in which the chain lies fixed in a plane rotating about the vertical through the upper support of the chain with constant angular velocity $\omega$.

This problem is governed by the singular boundary value problem

$$
\begin{gathered}
u^{\prime \prime}+\lambda u\left(u^{2}+s^{2}\right)^{-1 / 2}=0, \\
u(0)=0=u^{\prime}(1)
\end{gathered}
$$


where $u$ is the horizontal component of the tension and $\lambda$ is proportional to $\omega^{2}$. This problem does not admit closed-form solutions. Nevertheless, by cleverly combining the shooting method with Sturmian theory, Kolodner gave a detailed global description of all bifurcating branches (in terms of the nodal properties of $u$ ) and determined the location of the branches.

Kolodner's beautiful results inspired others to try his methods for different problems, but their success was limited. Pimbley $(1962,1963)$ was able to treat some related equations. On the other hand, I recall that E. L. Reiss noted that there was no obvious way that Kolodner's methods could handle boundary value problems for (2.1), especially when $B$ is not constant, whence (2.1) does not admit closed-form solutions. (The reason why Kolodner's methods handle (2.6), (2.7), but not (2.1), (2.4) devolves upon the difference in mathematical structure between the nonlinear terms $\sin \theta$ of $(2.1)$ and $u\left(u^{2}+s^{2}\right)^{-1 / 2}$ of (2.6).) The failure of Kolodner's global qualitative methods to handle some other apparently simple problems stimulated a number of mathematicians (most of whom were associated with New York University) to develop more effective methods for treating large classes of bifurcation problems. In this enterprise another problem from nonlinear elasticity, the axisymmetric buckling of a circular plate under a pressure applied to its edge, has played a central role. The plate theory employed was that of von Kármán. Let $\lambda$, representing the magnitude of the pressure, denote the eigenvalue parameter and $u$ denote the unknown function in this theory. Let $\lambda_{0}, \lambda_{1}, \ldots$ denote the eigenvalues of the equations linearized about $u=0$.

Friedrichs and Stoker (1941) used variational methods to show that everywhere on the bifurcating branch emanating from $\left(\lambda_{0}, 0\right)$, the function $u$ has no nodes. They showed that for any fixed value of $\lambda$ lying between $\lambda_{0}$ and $\lambda_{1}$ there are exactly three solutions, one of which is the trivial solution. They also used the method of (Lyapunov and) Schmidt to justify a perturbation expansion for the nontrivial branch of solutions emanating from the lowest eigenvalue of the linearized problem.

Keller, Keller and Reiss (1962) used the Poincaré shooting method to study the same problem. They showed that a nontrivial branch of solutions bifurcates from each point $\left(\lambda_{0}, 0\right),\left(\lambda_{1}, 0\right), \ldots$, that near each bifurcation point the branch lies to the right of the bifurcation point (as in Figure 2.5), and that on the intersection of the branch emanating from $\left(\lambda_{k, 0}\right)$ with a neighborhood of $\left(\lambda_{k, 0}\right)$, the function $u$ has exactly $k$ interior nodes. Reiss (1965) used similar methods to study the buckling of a spherical cap.

Detailed global results for this problem were finally obtained by Wolkowisky (1967). He proved that for each $\lambda>\lambda_{k}$ there are at least $k$ pairs of nontrivial solution pairs $\left(\lambda, \pm u_{j}\right), j=0, \ldots, k$, with $u_{j}$ having exactly $j$ internal nodes. To do this he used Sturmian theory to set up a solution operator for each $j \leqslant k$ and for each fixed $\lambda$. He was then able to use the Schauder Fixed Point Theorem to show that each operator has a fixed point with the requisite nodal properties. Wolkowisky (1969) extended his methods to handle a family of nonlinear Sturm-Liouville Problems. Wolkowisky's work is beautiful and clever. In retrospect, we may, however, observe that it does not say anything about the connectivity of the solution pairs he found. 
At the same time Berger (1967a) and Berger and Fife (1968) studied the semilinear system of partial differential equations governing the buckling of a von Kármán plate of any shape under a planar system of loads of magnitude $\lambda$. This problem has a natural energy functional $\varphi$. (I do not discuss these papers separately because their structure makes it inconvenient to do so.) Using the variational method of Lyusternik and Shnirel'man, Berger and Fife showed that on each energy surface $\{u: \varphi(u)=R\}, R \in(0, \infty)$, there is a countable family of distinct solution pairs. Moreover, they proved that exactly one nontrivial branch can bifurcate from a simple eigenvalue and they obtained partial results on the number of branches that can bifurcate from an eigenvalue of multiplicity 2 . (The delicate problem of correlating the number of nontrivial solution branches emanating from an eigenvalue of the linearized problem with the multiplicity of the eigenvalue is fundamental in bifurcation theory. For variational problems Krasnosel'skir (1953) obtained the first results (cf. Krasnosel'skiĭ (1956), Chapter 4), Berger (1967b, 1969) announced generalizations, and Böhme (1971), (1972) gave complete proofs along with some fascinating counterexamples.)

In the period from 1955 to 1970 and beyond, Vorovich had been independently developing a complete mathematical theory of boundary value problems for plates and shells of the von Kármán type. His results relied on functionalanalytic and topological techniques. Brief accounts of his contributions are given by Trenogin and Yudovich (1974, pp. 36, 127). It appears that a number of the results described above, obtained by American mathematicians, were found earlier by him. E.g., many of the results of Berger and Fife were announced by Vorovich (1958). According to Trenogin and Yudovich, Vorovich (1955) studied the local theory of the axisymmetric buckling of annular disks by the method of Lyapunov and Schmidt. (I have not been able to see a copy of this paper.) It thus appears that this work would have a clear-cut priority over that of Keller, Keller and Reiss (1962), were it not for the fact that the latter work treats the troublesome singularity at the center of the plate. The work of Vorovich (partially surveyed in his papers $(1969,1970)$ ) forms an important chapter in the application of functional-analytic and topological methods to nonlinear problems of physics. Unfortunately, western scientists with the sophistication to appreciate it seemed largely unaware of it. Consequently it had no influence on the next and crucial step of the development of global continuation methods of bifurcation theory.

Motivated primarily by the work of Kolodner and of Wolkowisky and to a lesser extent by that of Berger and Fife, Crandall and Rabinowitz (1970) studied global behavior of nonlinear Sturm-Liouville problems of a sort that includes (2.1), (2.4) and (2.6), (2.7). They developed effective methods to describe the nodal properties of solutions. In place of the Schauder Fixed Point Theorem used by Wolkowisky, they employed the Leray-Schauder degree. By exploiting its invariance under homotopy, Rabinowitz (1970) was able to show that branches of solution pairs of nonlinear Sturm-Liouville problems enjoy global connectivity and nodal properties (to be described shortly). At the same time Turner (1970) was able to show that branches of solution pairs of 
nonlinear integral equations with oscillation kernels have connected projections on the plane of parameter and norm. (These equations have nodal properties generalizing those of Sturm-Liouville problems.)

The basic parameter used by Wolkowisky is the eigenvalue parameter and that used by Berger and Fife is the prescribed value of a norm-like energy functional. If the bifurcating branches are know to be curves, then it is natural to give them an intrinsic parametrization. In general, however, bifurcating branches are merely connected. The approach of Crandall and Rabinowitz and of Rabinowitz may roughly be likened to one that relies on what would be a natural parametrization for branches that are curves.

The global ideas of Rabinowitz (1970) were simplified and generalized by Rabinowitz (1971). Crandall and Rabinowitz (1971) systematized and refined the local theory of bifurcation. (Perhaps the most accessible source of these results is the paper of Rabinowitz (1973).) The fundamental theorem of Rabinowitz (1971) is this:

2.8. Theorem. Let $\mathscr{B}$ be a Banach space with norm $\|\cdot\|$. Let $L: \mathscr{B} \rightarrow \mathscr{B}$ be compact and linear. Let $F: \mathbf{R} \times \mathscr{B} \rightarrow \mathscr{B}$ be compact and continuous with $F(\lambda, u)$ $=o(\|u\|)$ as $u \rightarrow 0$, uniformly for $\lambda$ in bounded intervals. Let $\mathcal{S}$ be the closure of nontrivial solution pairs of

$$
u=\lambda L u+F(\lambda, u)
$$

in $\mathbf{R} \times \Re$. If $\mu$ is an eigenvalue of odd algebraic multiplicity of the linearization

$$
v=\mu L v
$$

of (2.9), then $\mathcal{S}$ contains a maximal, closed, connected subset $\mathcal{C}(\mu)$ that contains $(\mu, 0) . \mathcal{C}(\mu)$ has at least one of the following two properties: (i) $\mathcal{C}(\mu)$ is unbounded in $\mathbf{R} \times \Re$; (ii) $\mathcal{C}(\mu)$ contains a point $(\nu, 0)$ where $\nu$ is another eigenvalue of (2.10).

Using this theorem together with some results of Crandall and Rabinowitz (1971) we can readily obtain a detailed global description of all bifurcating branches of (2.1), (2.4). We sketch the details. We integrate (2.1) twice, accounting for (2.4), to obtain

$$
\theta(s)=\lambda \int_{0}^{s} B(t)^{-1} \int_{t}^{1} \sin \theta(r) d r d t
$$

We take the Banach space of Theorem 2.8 to be $C^{1}([0,1])$. By writing $\sin \theta=\theta+(\sin \theta-\theta)$ in (2.11) we readily convert (2.11) to the form (2.9). A simple application of the Arzelà-Ascoli Theorem shows that the $L$ and $F$ thereby induced have the requisite compactness. Moreover, the linear integral equation corresponding to (2.10) is equivalent to (2.3) subject to boundary conditions of the form (2.4). The Sturmian Theory says that the eigenvalues $\lambda_{0}, \lambda_{1}, \ldots$ of the linearized problem are simple, positive, and unbounded and that they can be ordered thus: $0<\lambda_{0}<\lambda_{1}<\cdots$ with the eigenfunction $\psi_{k}$ corresponding to $\lambda_{k}$ having exactly $k$ zeros on $(0,1)$, each of which is simple. Now let $\mathcal{G}_{k}$ be the set of all functions $\theta$ in $C^{1}([0,1])$ satisfying (2.4) and having exactly $k+1$ zeros $s_{0}, s_{1}, \ldots, s_{k}$ on $[0,1]$, with $0=s_{0}<s_{1}<\cdots<s_{k}<1$ and 
with $\theta^{\prime}\left(s_{j}\right) \neq 0$ for $j=0,1, \ldots, k \cdot \mathcal{G}_{k}$ is open in $C^{1}([0,1])$ (but not in $C^{0}([0,1])$ ). A theorem of Crandall and Rabinowitz (1971) says that if $(\lambda, \theta)$ is a solution of (2.11) lying close enough to $\left(\lambda_{k}, 0\right)$, then $\theta$ is approximated in $C^{1}([0,1])$ by a multiple of $\psi_{k}$, whence $\theta \in \mathcal{G}_{k}$. We now assert that $\mathcal{C}\left(\lambda_{k}\right) \backslash[\mathbf{R} \times\{0\}] \subset \mathcal{G}_{k}$. If not, there would be a $(\bar{\lambda}, \bar{\theta}) \in \mathcal{C}\left(\lambda_{k}\right) \backslash[\mathbf{R} \times\{0\}]$ with $\bar{\theta} \in \partial \mathcal{S}_{k}$. But members of $\partial \mathcal{S}_{k}$ have double zeros. Thus $\bar{\theta}$ would satisfy an initial value problem for (2.1) subject to initial conditions of the form $\bar{\theta}(\sigma)=0=\bar{\theta}^{\prime}(\sigma)$. The unique solution of this problem is $\bar{\theta}=0$, a contradiction. Since $\psi_{j} \in \mathcal{C}\left(\lambda_{j}\right), j=$ $0,1,2, \ldots$, the branch $\mathcal{C}\left(\lambda_{k}\right)$ cannot contain $\left(\lambda_{l}, 0\right)$ with $l \neq k$, for if so, there would be a pair $(\lambda, \theta)$ in $\mathcal{C}\left(\lambda_{k}\right) \cap \mathcal{C}\left(\lambda_{l}\right) \backslash[\mathbf{R} \times\{0\}] \subset \mathcal{G}_{k} \cap \mathcal{S}_{l}=\varnothing$. Thus Theorem 2.8 implies that each branch $\mathcal{C}\left(\lambda_{k}\right)$ is unbounded in $\mathbf{R} \times C^{1}([0,1])$ and that the nodal properties of $\theta$ are preserved along each branch. Suitable estimates (cf. Crandall and Rabinowitz (1970)) show that the branches are disposed as in Figure 2.5.

While other global methods of bifurcation theory, such as the theory of Lyusternik and Shnirel'man, can be applied to this problem, the methods of Crandall and Rabinowitz yield the most detailed information about the bifurcating branches. These same methods can handle Kolodner's problem (cf. Stuart (1975)).

There have been numerous refinements of Theorem 2.8. Rabinowitz (1970) observed that the $\lambda L$ in (2.9) could be replaced with $L(\lambda)$. Ize (1976) and Magnus (1976) developed the treatment of such operators. Dancer (1973) treated problems in which the $F$ in (2.9) is real analytic. Nussbaum (1975) and McLeod and Turner (1976) developed methods to handle bifurcation problems in which (2.9) is replaced by an equation involving nondifferentiable operators. Problems in which the hypotheses on the compactness of $L$ and $F$ in (2.9) are replaced by weaker assumptions were analyzed by Stuart (1973), Toland (1976), and Alexander and Fitzpatrick (1979). An extensive bibliography is given by Alexander (1981). These theories have been applied by Antman and Rosenfeld (1978) and Antman (1978) to other problems of nonlinear elasticity (including theories for plates that do not suffer the manifold defects of von Kármán's theory, not the least of which is that his equations are not valid for the large deformations studied in global analyses).

A generalization of the planar buckling problem for a rod under terminal thrust, described by (2.1) and (2.4), is that of the spatial buckling of a rod under terminal thrust and twist. Greenhill (1883) analyzed his ineptly formulated eigenvalue problem for the linearized equations for this problem. Properly formulated linear boundary value problems for the rod have nontrivial solutions when the pair $(\lambda, \mu)$ of parameters lie on any of a countable family of eigencurves. One might expect that nontrivial solution pairs of nonlinear versions of Greenhill's problem lie on "two-dimensional sheets". It is clear that the methods of Rabinowitz can be applied to this problem by freezing one of the parameters. But it is also clear that the resulting information would be unsatisfactorily incomplete: E.g., one could show that the nontrivial solution pairs lie on unions of one-dimensional connected sets, but could not assert how these one-dimensional connected sets are attached. To circumvent this difficulty Alexander and Antman (1981) used the theory of 
Čech cohomology to obtain easily verifiable conditions ensuring that bifurcating from a given point on an eigensurface of an $n$-parameter problem is a connected set of solution pairs each point of which has Lebesgue dimension $\geqslant n$. (Related results have been obtained by Fitzpatrick, Massabò and Pejsachowicz (1983).) This theory, a natural generalization of that of Rabinowitz, was then applied to treat general nonlinear analogs of Greenhill's problem, for which it was expressly designed, by Antman and Kenney (1981).

Euler (1780) formulated the problem for the buckling of a column under its own weight and computed the lowest buckling load for a uniform column. Oblivious of this work, Greenhill (1881) used available results on Bessel functions to compute the same buckling load. Despite his advantage of one century of scientific progress, Greenhill's formulation was inferior to that of Euler in precision, method, and insight. Moreover, Greenhill must suffer the posthumous embarrassment of having computed a buckling load less accurate than Euler's. (Cf. Truesdell (1960, p. 363).)

If the column is not uniform, then the mass density per unit length may be regarded as an eigenvalue parameter, which is infinite-dimensional. Alexander and Antman (1983) extended their results of 1981 for a finite number of parameters to handle this case, and then used the resulting theory to obtain a detailed global analysis of this buckling problem.

3. The peculiarities of nonlinear elasticity. To describe other influences of nonlinear elasticity on analysis, it is first necessary to describe the mathematical structure of nonlinear elasticity.

A material point of a body may be identified with the position $x$ it occupies in a reference configuration of the body; the body itself may then be identified with the region $\Omega$ it occupies in this reference configuration. The position of material point $x$ at time $t$ is denoted $p(x, t)$. It is taken to be an element of the Euclidean 3-space $\mathbf{E}^{3}$. The theory of continuum mechanics yields a set of differential equations for $p$ that reflect the mechanical properties of $\Omega$ and the environment in which it is placed.

It is clear to anyone who has observed the effect of spiked shoes on a wooden floor that force intensity per unit area is a more useful variable for describing deformations than force itself. If $x$ is a material point in the interior of a body $\Omega$ undergoing a motion $p$ and if $n$ is a unit vector, then the intensity of contact force at $x$ per unit reference area of the plane $\{y \in \Omega:(y-x) \cdot n$ $=0\}$ exerted by the material of $\{y \in \Omega:(y-x) \cdot n \geqslant 0\}$ on that of $\{y \in \Omega$ : $(y-x) \cdot n<0\}$ at time $t$ has the form $T(x, t) n$, where $T(x, t)$ is a tensor, i.e., a linear transformation from $\mathbf{E}^{3}$ to itself (called the first Piola-Kirchhoff stress tensor at $(x, t))$. (This representation for the contact force was discovered by Cauchy.) The inner-product space of these tensors is denoted $\mathcal{L}$. Under favorable conditions of regularity, the requirement that the resultant force on each part of a body equal the time rate of change of linear momentum of that part yields the equation of motion

$$
\nabla \cdot T^{*}+f=\rho \frac{\partial^{2} p}{\partial t^{2}} .
$$


Here $\nabla \cdot$ denotes the divergence operator (with respect to $x$ ), the asterisk denotes transpose, $f(x, t)$ represents the given force per unit reference volume at $(x, t)$, and $\rho(x)$ is the given mass density at $x$ in the reference configuration. Mechanical properties of materials are characterized by relations between $T(x, t)$ and the history of $p$ up to time $t$ in a neighborhood of $x$. E.g., we can characterize the mechanical response of a rubber band by giving an expression for the force per unit area needed to effect a given change of length. The material at $x$ is elastic if there is a function $S$ such that

$$
T(x, t)=S\left(\frac{\partial p}{\partial x}(x, t), x\right) .
$$

Here $\partial p / \partial x$ represents the derivative of $p$. (It is the linear transformation whose matrix consists of the derivatives of the components of $p$ with respect to the components of $x$.) Equation (3.2) specifically prevents the material from exhibiting viscous (frictional) effects, which could be accounted for by allowing $S$ to depend on the velocity gradient $\partial^{2} p / \partial x \partial t$ as well. Since $S$ depends only on the present value of $\partial p / \partial x$, no account is taken of the influence of the past history of the deformation on the present value of the stress. The actual form of (3.2) must be specialized somewhat to ensure that material properties are unaffected by rigid motions. The requirement that the resultant torque on each part of a body equal the time rate of change of angular momentum of that part yields a relation that the use of $(3.1)$ reduces to

$$
T\left(\frac{\partial p}{\partial x}\right)^{*}=\left(\frac{\partial p}{\partial x}\right) T^{*}
$$

We account for this condition by requiring the function $S$ to satisfy

$$
S(G, x) G^{*}=G S(G, x)^{*}
$$

identically for all tensors $G$ with a positive determinant.

If we now substitute (3.2) into (3.1), we obtain a quasilinear system of partial differential equations for $p$, which we can supplement with initial and boundary conditions. What distinguishes this system from such systems in general, such as those describing electromagnetic effects in rigid media? The most obvious answer is that the unknown $p$ has direct geometric significance: It describes a class of mappings of regions of $\mathbf{E}^{3}$ into $\mathbf{E}^{3}$. Consequently, continuum mechanics has within it the richness and complexity of the geometry of $\mathbf{E}^{3}$. This geometry is a fundamental source of difficulty for mechanics. E.g., since $p(\cdot, t)$ describes the deformation of a material body, we require that it be one-to-one so that two distinct material points cannot simultaneously occupy the same point in space. But this requirement of injectivity is a global restriction on the function $p$, which is otherwise described locally by (3.1), (3.2), and side conditions. Techniques capable of handling the injectivity of $p$ would doubtless yield solutions of major unsolved problems of geometry. Moreover, the serious consideration of the physical aspects of this question would require the prescription of a strategy for stating boundary conditions that are to hold if two distinct parts of the boundary come into contact.

These difficulties force us to contemplate the more modest requirement that $p(\cdot, t)$ preserve orientation, which is equivalent to the requirement that the 
local ratio of volume in the configuration at time $t$ to that of the reference volume be everywhere positive. If $p(\cdot, t)$ is continuously differentiable, then this condition is expressed by

$$
\operatorname{det}(\partial p / \partial x)>0
$$

This of course is a purely local restriction. We can incorporate it into initial-boundary value problems in a natural way by restricting $S(\cdot, x)$ to

$$
\mathfrak{L}^{+} \equiv\{G \in \mathcal{L}: \operatorname{det} G>0\},
$$

and by requiring that

$$
|S(G, x)| \rightarrow \infty \quad \text { as det } G \rightarrow 0 .
$$

Thus the violation of (3.5) would be signaled by the failure of $p$ to be a regular solution of (3.1), (3.2).

The modern theory of differential equations tells us that it is foolhardy to seek classical solutions of problems for (3.1), (3.2) because (i) there may not be any, and (ii) even if they exist, it is easier to find them by seeking solutions in a larger class of functions and then proving that such solutions are necessarily smoother than other members of the class. In particular, the available theory suggests that to solve a boundary value problem for the static version of (3.1), (3.2) (in which $p$ is independent of $t$ ), one should first pose the problem in a weak form and then seek solutions in a function space like the Sobolev space $W^{1, q}(\Omega)$. (The actual space chosen is dictated by the form of $S(G, x)$ for large $|G|$.) If $x \mapsto p(x)$ belongs to such a space, then (3.5) should be relaxed to hold almost everywhere. But in this case we might have difficulty in defining $\operatorname{det}(\partial p / \partial x)$ because this is the sum of products of derivatives of $p$.

A more serious problem is that the set of all $p$ 's satisfying (3.5) is not convex. (If $\Omega$ is not simply-connected, this set may even consist of a countable disjoint union of nonconvex sets.) Thus the large body of results of nonlinear analysis concerned with convex sets is not readily available to handle problems of nonlinear elasticity.

It is clear that both the mechanical response of an elastic material and the mathematical classification of the system (3.1), (3.2) devolve exclusively on the form of $S$. As a primitive requirement on $S$ we might demand that pulling a specimen of material in one direction results in a lengthening rather than a shortening of the material in that direction. It is not obvious how to translate this requirement into a precise and physically valid mathematical statement because there are different kinds of stresses that can measure the amount of pull, there are different kinds of strains to measure the elongation, and the pulling produces not only an elongation in the direction of pull, but also contractions in the transverse directions. One might be led by mathematical optimism to propose that $S(\cdot, x)$ be strictly monotone in the sense that

$$
[S(G+H, x)-S(G, x)]: H>0 \quad \forall G
$$

and

$$
\forall H \neq 0 \text {. }
$$


Here ":" represents the inner product on the space of tensors. If $S(\cdot, x)$ is differentiable, then a slightly stronger restriction is that

$$
H: \frac{\partial S}{\partial G}(G, x): H>0 \quad \forall G
$$

subject to (3.9). If $S(\cdot, x)$ is everywhere defined (i.e., if (3.5) is ignored) and if $S(\cdot, x)$ satisfies $(3.8),(3.9)$ and suitable growth conditions, then the analysis of boundary value problems for the static version of $(3.1),(3.2)$ is in a relatively good state: Weak solutions exist, are unique, and possess certain regularity properties (cf. Giaquinta (1983) and Giusti (1983)). The apparent virtue of yielding uniqueness is actually fatal for (3.8) and (3.9) because it means that however thin a rod is and however large the thrust applied to it is, it can never buckle. One of the main motivations for suffering the difficulties of nonlinear elasticity is to be able to describe such buckling phenomena.

If $S(\cdot, x)$ is defined only on $\mathfrak{L}^{+}$, then another objection to (3.8) and (3.9) arises: If $S$ simultaneously satisfies (3.7)-(3.9), then the nonconvexity of $\mathfrak{L}^{+}$ would require $S$ to have very special, indeed pathological forms. In fact, $S(\cdot, x)$ could not be the gradient of a scalar $\Phi(\cdot, x)$ on $\mathcal{L}^{+}$with $\Phi(F, x) \rightarrow \infty$ as det $F \rightarrow 0$. (Other serious objections to (3.8) and (3.9) are discussed by Ball (1977b).)

The easiest way to allow boundary value problems for static versions to admit multiple solutions is to let $S$ depend on $p$ as well as $\partial p / \partial x$, while (3.8) and (3.9) still hold. The resulting equations can be handled by the theory of pseudo- or semi-monotone operators. But this adjustment would imply that the material properties of the body would depend upon its position, and would thus be physically unacceptable.

A condition weaker than (3.8), (3.9) is (the strict form of) the strong ellipticity condition: (3.8) holds

\section{$\forall H$ of rank 1 .}

(If $S$ is differentiable, a slightly stronger version of this condition is that (3.10) holds for (3.11).) This condition does ensure that an elongation accompanies a pull and that (3.1), (3.2) is hyperbolic. This means that (3.1), (3.2) can have solutions with a full range of wave-like behavior. Moreover, the requirements that (3.8), (3.11) hold, that det $G>0$, and that $|S(G, x)| \rightarrow \infty$ as det $G \rightarrow 0$ are compatible, because the restriction (3.8) to hold just for tensors of rank 1 perfectly matches the fact that det $G$ is an affine function of each of its entries when the other entries are held fixed. (Despite these virtues of the strong ellipticity condition, Ericksen (1983) has made a persuasive case that it does not capture effects observed in certain real crystalline solids. Thus the strong ellipticity condition should not be regarded as universally valid.)

The theory of strongly elliptic, quasilinear systems of partial differential equations is unfortunately in a primitive state. By embracing the strong ellipticity condition as a reasonable restriction for elastic materials, we might seem to retard the rate of progress in elasticity to that of such systems. In fact, as we observe in the next section, the joining of these two disciplines has led to new progress both in the theory of quasilinear elliptic systems and in other 
fields of analysis. Despite its peculiarities, nonlinear elasticity merits the attention of analysts because it is the most accessible physical theory described by quasilinear systems.

A detailed discussion of the questions sketched in this section is given by Ball (1977b) and Antman (1983).

4. Calculus of variations. Weak convergence. If there is a function $\Phi: \mathfrak{L}^{+} \rightarrow \mathbf{R}$ such that

$$
S(G, x)=\frac{\partial \Phi}{\partial G}(G, x),
$$

then the material of $\Omega$ is said to be hyperelastic. If (4.1) holds, if $f=0$ (for simplicity), and if $p$ is independent of $t$, then (3.1), (3.2) is equivalent to the Euler-Lagrange equations for the functional

$$
p \mapsto \int_{\Omega} \Phi\left(\frac{\partial p}{\partial x}(x), x\right) d v(x)
$$

where $d v$ is the differential volume. We may then contemplate studying boundary value problems for (3.1), (3.2) by the direct methods of the calculus of variations. In this case a sufficiently smooth minimizer of (4.2) satisfying appropriate side conditions would be a solution of a boundary value problem for (3.1), (3.2).

Ball (1977a, b) showed the existence of a minimizer of (4.2) in spaces like the Sobolev space $W_{0}^{1, q}(\Omega)$ by adapting the deep, direct methods of Morrey (1952, 1966) to handle the difficulties associated with (3.5)-(3.7). In this process, Ball introduced important new ideas into the calculus of variations. These ideas in turn inspired further developments in other areas of analysis.

Recall that $\Phi(\cdot, x)$ defined on $\mathcal{L}$ is convex if

$$
\begin{aligned}
\Phi(\lambda G+(1-\lambda) H, x) \leqslant \lambda \Phi(G, x)+(1-\lambda) \Phi( & H, x) \\
& \forall \lambda \in[0,1], \forall G
\end{aligned}
$$

and

$\forall H$.

If the inequality of (4.3) is replaced by a strict inequality, if $\lambda$ is restricted to $(0,1)$, and if $(4.1)$ holds, then this modified version of $(4.3),(4.4)$ is equivalent to (3.8), (3.9). If $\Phi(\cdot, x)$ is defined only on $\varrho^{+}$, then we may say that $\Phi(\cdot, x)$ is convex on $\mathcal{L}^{+}$if the restriction of $\Phi$ to each closed line segment of $\mathcal{L}^{+}$is convex. $\Phi(\cdot, x)$ is said to satisfy the (generalized) Legendre-Hadamard condition on $\mathrm{L}^{+}$ if (4.3) holds

(4.5) $\forall H$ of rank 1 for which the line segment joining $G$ and $H$ lies in $\varrho^{+}$.

(If the inequality of (4.3) is replaced by a strict inequality, if $\lambda$ is restricted to $(0,1)$, and if $(4.1)$ holds, then this modified version of $(4.3),(4.5)$ is equivalent to (3.8), (3.11).) Let $\Re$ be an open subset of $\mathcal{L}$. In the terminology of Morrey $(1952,1966), \Phi(\cdot, x)$ is said to be strongly quasiconvex on $\Re$ if

$$
\int_{D} \Phi\left(G+\frac{\partial u}{\partial y}(y), x\right) d v(y) \geqslant \Phi(G, x) v(D)
$$


$\forall G \in \Re, \forall$ bounded, open $D \in \mathbf{E}^{3}$, and $\forall u \in C_{0}^{\infty}(D)$ for which $G+$ $\partial u(y) / \partial y \in \mathfrak{R}$ when $y \in D$. Here $v$ is the volume. (Carefully note the arguments in the integrand of (4.6).) Using this condition, Morrey established the existence of minimizers for functionals of the form (4.2) on Sobolev spaces when $\Phi(\cdot, x)$ is strongly quasiconvex on $\mathcal{L}$. This remarkable result was the first to give promise of treating quasilinear elliptic systems of the sort that arise in the theory of elasticity. Morrey's theory is incapable of handling (3.5) and (3.7), however.

To construct a theory without these defects, Ball introduced the concept of polyconvexity. Let $G^{\times}$denote the tensor of cofactors of $G$. (If $G$ is invertible, then Cramer's rule says that $G^{\times}=(\operatorname{det} G)\left(G^{-1}\right)^{*}$ where the star denotes the transpose.) $\Phi(\cdot, x)$ is said to be polyconvex on $\mathcal{L}^{+}$if there is a convex function $\Psi(\cdot, \cdot, \cdot, x)$ on $\mathcal{L} \times \mathcal{L} \times(0, \infty)$ such that

$$
\Phi(G, x)=\Psi\left(G, G^{\times}, \operatorname{det} G, x\right) .
$$

Note that the domain of $\Psi(\cdot, \cdot, \cdot, x)$ is a half-space, whereas that of $\Phi(\cdot, x)$ is not even convex. The following chain of implications holds:

$$
\begin{aligned}
& \left\{\Phi(\cdot, x) \text { is convex on } \mathfrak{L}^{+}\right\} \\
& \quad \Rightarrow\left\{\Phi(\cdot, x) \text { is polyconvex on } \mathfrak{L}^{+}\right\} \\
& \quad \Rightarrow\left\{\Phi(\cdot, x) \text { is strongly quasiconvex on } \mathcal{L}^{+}\right\} \\
& \quad \Rightarrow\left\{\Phi(\cdot, x) \text { satisfies the Legendre-Hadamard condition on } \mathfrak{L}^{+}\right\} .
\end{aligned}
$$

The first two implications, found by Ball, are not equivalences. It is not known whether the last implication, found by Morrey, is an equivalence. Ball showed that the assumption of polyconvexity is not so severe as to preclude multiple solutions of the equilibrium equations. Moreover, he showed that a variety of accepted models of elastic response can be characterized by polyconvex $\Phi$ 's.

To appreciate the mathematical importance of the notion of polyconvexity and the supporting ideas of weak convergence let us recall the basic theorem of the calculus of variations, which can be traced back to Weierstrass.

4.9. THEOREM. A sequentially weakly lower semicontinuous functional on a bounded, weakly closed, nonempty subset of a reflexive Banach space attains its minimum there.

(A proof is given by Vainberg (1956), e.g.) A functional $\varphi$ is sequentially weakly lower semicontinuous if

$$
\varphi(u) \leqslant \liminf \varphi\left(u_{k}\right) \quad \text { as } u_{k} \rightarrow u .
$$

Here the half arrow denotes weak convergence on the Banach space to which the domain of $\varphi$ belongs. Available theorems ensuring that functionals $\varphi$ satisfy (4.10) rely on certain convexity properties of $\varphi$.

To study the sequential weak lower semicontinuity of (4.2) on a subset of $W^{1, q}(\Omega)$ with $q>1$ when $\Phi(\cdot, x)$ is polyconvex on $\mathcal{L}^{+}$, we need to know the behavior of the sequences $\left(\partial p_{k} / \partial x\right)^{\times}$and $\operatorname{det}\left(\partial p_{k} / \partial x\right)$ as $p_{k} \rightarrow p$ in $W^{1, q}(\Omega)$. That this behavior can be easily characterized and that $\Psi(\cdot, \cdot, \cdot, x)$ is convex are the underlying mathematical justifications of polyconvexity. Among the 
basic results on weak convergence that Ball obtained are the following:

4.11. TheOREM. Let $\phi: \mathcal{L} \rightarrow \mathbf{R}$ be continuous. If $\phi$ is a null Lagrangian, i.e., if

$$
\int_{\Omega} \phi\left(\frac{\partial p}{\partial x}(x)+\frac{\partial u}{\partial x}(x)\right) d v(x)=\int_{\Omega} \phi\left(\frac{\partial p}{\partial x}(x)\right) d v(x),
$$

for all $p \in C^{1}(\bar{\Omega})$ and for all $u \in C_{0}^{\infty}(\Omega)$, then $\phi(G)$ must be an affine function of $\left(G, G^{\times}, \operatorname{det} G\right)$.

4.13. THEOREM. Let $q \geqslant 1$ and $C \geqslant 0$. Let $\phi: \mathcal{L} \rightarrow \mathbf{R}$ be continuous and satisfy $|\phi(G)| \leqslant C\left(1+|G|^{q}\right)$. If the function $W^{1, q}(\Omega) \ni p \mapsto \phi(\partial p(\cdot) / \partial x) \in L^{1}(\Omega)$ is continuous from the weak topology of $W^{1, q}(\Omega)$ to the weak topology of $L^{1}(\Omega)$, i.e., if

$$
\phi\left(\frac{\partial p_{k}}{\partial x}(\cdot)\right) \rightarrow \phi\left(\frac{\partial p}{\partial x}(\cdot)\right) \quad \text { in } L^{1}(\Omega) \text { as } p_{k} \rightarrow p \text { in } W^{1, q}(\Omega),
$$

then $\phi$ is a null Lagrangian.

F. Murat pointed out to me that there is a more convenient variant of this theorem in which the weak topology of $L^{1}$ is replaced with the pseudo-topology of distributions, or equivalently, with the vague topology of measures, in which case (4.14a) is replaced with

$$
\begin{gathered}
\int_{\Omega} \phi\left(\frac{\partial p_{k}}{\partial x}(x)\right) \psi(x) d v(x) \rightarrow \int_{\Omega} \phi\left(\frac{\partial p}{\partial x}(x)\right) \psi(x) d v(x) \\
\forall \psi \in \mathcal{Q}(\Omega) \text { as } p_{k} \rightarrow p \text { in } W^{1, q}(\Omega) .
\end{gathered}
$$

These results show that those $\phi$ 's satisfying the hypotheses of Theorem 4.13 are exactly the functions of $G$ that appear in the arguments of $\Psi$ in (4.7). To show that these functions $\phi$ actually satisfy the hypotheses of Theorem 4.13 for suitable $q$, Ball exploited the fact that both $(\partial p / \partial x)^{\times}$and $\operatorname{det}(\partial p / \partial x)$ can be written as divergences and can thereby be given meaning in the sense of distributions. These results and the convexity of $\Psi(\cdot, \cdot, \cdot, x)$ enabled Ball to invoke a general lower semicontinuity theorem of Ekeland and Temam (1972) to show that (4.2) is minimized when $\Psi$ satisfies suitable growth conditions. Since an incompressible material is characterized by the constraint $\operatorname{det}(\partial p / \partial x)$ $=1$, Ball was able to use his apparatus to minimize a corresponding energy for such materials. Technical difficulties with (3.5) and (3.7) have so far prevented the appearance of a proof asserting that these minimizers are weak solutions of the Euler-Lagrange equations. Ball $(1980,1982)$ has nevertheless obtained a variety of regularity results that illuminate the hypotheses we have discussed.

In summary, Ball was the first and only one to make progress toward an effective global existence theory for the partial differential equations of elastostatics under physically reasonable assumptions on the material response. (There are detailed global results for ordinary differential equations of elasticity, detailed local results for three-dimensional static and dynamic problems, and global results for static problems in which the models used do not account for the characteristic difficulties discussed in §3.) Ball's work on these problems contributed significantly to the methods of the calculus of variations and 
of quasilinear systems. His treatment of problems of weak convergence of composite functions inspired important and useful developments, which we now discuss.

A basic method for demonstrating the existence of solutions of nonlinear partial differential equations is to construct solutions to a sequence of approximating problems and then to use theorems on weak compactness in ways that exploit the structure of the nonlinear operators in order to show that a subsequence of the approximating solutions converges to a solution of the actual problem. Such methods were intensively cultivated in the 1960's and 1970's. (Cf. Lions (1969).) An important role in this development was played by the theory of operators of monotone type. Although these methods enjoyed great success, there were many problems that proved to be resistant to them. One difficulty hinged on the lack of effective characterizations of weak limits of composite functions. In the mid 1970's Murat and Tartar began to study weak convergence with the intent of developing methods capable of handling otherwise intractable problems.

To appreciate their task, let us first consider a classical result. Let $\Omega$ be an open subset of $\mathbf{R}^{N}$. Let $u^{n}=\left(u_{1}^{n}, \ldots, u_{N}^{n}\right)$ and $v^{n}=\left(v_{1}^{n}, \ldots, v_{N}^{n}\right)$ be vector-valued distributions over $\Omega: u^{n}, v^{n} \in\left(\mathscr{Q}^{\prime}(\Omega)\right)^{N}$. Suppose that $\left\{u^{n}\right\}$ is confined to a bounded set of $\left(W^{1,2}(\Omega)\right)^{N}$, that $\left\{v^{n}\right\}$ is confined to a bounded set of $\left(L^{2}(\Omega)\right)^{N}$, and that $u^{n} \rightarrow u$ and $v^{n} \rightarrow v$ in $\left(L^{2}(\Omega)\right)^{N}$. Then the compact embedding theorem of Rellich and Kondrashov implies that

$$
\sum_{j=1}^{N} u_{j}^{n} v_{j}^{n}-\sum_{j=1}^{N} u_{j} v_{j} \text { in } \mathscr{Q}^{\prime}(\Omega) .
$$

The most primitive generalization of this theorem, inspired by problems of homogenization, is

4.16. Div-Curl Theorem (Murat (1978)). Let $u^{n}, v^{n} \in\left(\mathscr{Q}^{\prime}(\Omega)\right)^{N}$, let

$$
\left\{\sum_{k=1}^{N} \frac{\partial u_{k}^{n}}{\partial x_{k}}, \frac{\partial v_{i}^{n}}{\partial x_{j}}-\frac{\partial v_{j}^{n}}{\partial x_{i}}, i, j=1, \ldots, N\right\}
$$

be confined to bounded sets of $L^{2}(\Omega)$, and let $u^{n} \rightarrow u$ and $v^{n} \rightarrow v$ in $\left(L^{2}(\Omega)\right)^{N}$. Then (4.15) holds (even though $u_{1}^{n} v_{1}^{n}$, say, need not converge weakly to $u_{1} v_{1}$ in $\left.\mathscr{Q}^{\prime}(\Omega)\right)$.

A comparison of the hypotheses of Theorem 4.16 with those of the preceding paragraph shows why J.-L. Lions termed the new theory "Compensated Compactness".

The first set of results, containing Theorem 4.16, were obtained by Murat and Tartar in 1974. In 1975, Ball showed Tartar his results on variational problems from elasticity. Murat and Tartar then realized that compensated compactness could be expanded into a far richer theory capable of subsuming Ball's results. Included in this theory are major extensions of Theorem 4.16 to account for far more general complementary conditions on $u^{n}$ and $v^{n}$ and to account for other nonlinear functions of $u$ and $v$ besides their scalar product. (Cf. Murat (1981), Tartar (1979).) 
A powerful tool in the construction of a unified theory was the following result of Tartar (1979), based on ideas of L. C. Young and McShane.

4.17. TheOREM. Let $K \subset \mathbf{R}^{M}, \Omega \subset \mathbf{R}^{N}$ be bounded and open. Let $f: \mathbf{R}^{M} \rightarrow \mathbf{R}$ be continuous. Let $u_{n}: \Omega \rightarrow \mathbf{R}^{M}$ be such that $u_{n}(x) \in K$ for almost all $x$ in $\Omega$. Then there exists a subsequence $\left\{u_{n}\right\}$ of $\left\{u_{n}\right\}$ (independent of $f$ ) and a family of probability measures $\left\{\nu_{x}, x \in \Omega\right\}$ with supp $\nu_{x} \subset K$ such that

$$
f\left(u_{n}(\cdot)\right) \stackrel{*}{\rightarrow} F(\cdot) \quad \text { in } L^{\infty}(\Omega) \text { with } F(x) \equiv \int_{\mathbf{R}^{M}} \nu_{x}(y) f(y) d y .
$$

Conversely, if $\nu$ has these properties, then there is a sequence $u_{n}: \Omega \rightarrow \mathbf{R}^{M}$ with $u_{n}(x) \in K$ for almost all $x$ in $\Omega$ such that (4.18) holds for all continuous $f$ : $K \rightarrow \mathbf{R}$.

A corollary of this result, suggesting the range of its usefulness, is

4.19. Corollary. Let $u_{n} \stackrel{*}{\rightarrow} u$ in $L^{\infty}(\Omega)$. Then $u_{n} \rightarrow u$ strongly in $L^{p}(\Omega)$ for $p \in(1, \infty)$ if and only if $\nu_{x}$ is the Dirac delta $\delta_{u(x)}$ supported at $u(x)$.

Tartar (1979) applied compensated compactness to treat first-order nonlinear hyperbolic conservation laws by using entropy conditions. Tartar was well aware that the results he obtained were by then classical: The importance of his work lie in the novelty of his method, a method with promise for treating more recalcitrant problems. This promise was recently realized in the work of DiPerna (1983, 1984), who obtained new existence results for Cauchy problems for second-order systems of conservation laws for large data. DiPerna also obtained the first convergence theorem for finite difference approximations of the solutions of such systems. We note that such systems describe not only gas dynamics, but also one-dimensional models of nonlinear elasticity. Thus a theory developed under the stimulus of one class of problems from nonlinear elasticity has illuminated another class.

A practical virtue of the theory of compensated compactness is that it reduces the number of a priori estimates needed in a given proof. It thereby yields proofs for problems where the estimates, formerly deemed crucial, are not available. Besides in the references listed above, systematic presentations of the theory and its applications, together with new developments have been given by Dacorogna (1982) and Tartar (1983). It is of course too early to see whether compensated compactness will turn out to have far reaching implications or to be a tool of limited utility. Even if the latter happens, its successes to date are nevertheless of considerable importance.

5-1. Variational inequalities. Signorini (1959) posed the problem, now bearing his name, that in simplest terms is to determine the displacements in a heavy, linearly elastic body resting on a rigid, frictionless horizontal plane. The essential difficulty of this problem is that the region of contact between the body and the plane is not known a priori. It is conceivable that the contact set could be especially complicated.

Fichera (1964) was the first to study the existence and uniqueness of solutions to this problem, which is nonlinear because position fields satisfying 
the governing equations are subjected to a unilateral constraint that restricts their values to lie in a half space. Now the position field for Signorini's problem (for a nonlinearly elastic material) may be characterized as the minimizer $p$ of the potential energy functional (cf. (4.2))

$$
\int_{\Omega} \Phi\left(\frac{\partial p}{\partial x}, x\right) d v-\int_{\Omega} \rho g k \cdot p d v
$$

on the convex set

$$
\mathscr{K}=\{p: p(x) \cdot k \geqslant 0, x \in \partial \Omega\} .
$$

Here $k$ is the unit vector pointing in the upward direction and $g$ is the acceleration of gravity.

To see the issues involved in this variational problem, we study its simplest analog, namely to minimize the function

$$
\mathscr{K} \equiv[0, \infty] \ni u \mapsto \psi(u) \in \mathbf{R} .
$$

If $\psi$ is continuously differentiable and has a minimum at $v$ in $\mathcal{K}$, then

$$
\psi^{\prime}(v)=0 \quad \text { if } v>0, \quad \psi^{\prime}(v) \geqslant 0 \quad \text { if } v=0 .
$$

These restrictions can be unified into a single statement

$$
\psi^{\prime}(v) \cdot(w-v) \geqslant 0 \quad \forall w \in \mathscr{K},
$$

which in turn can be readily generalized to a Banach space setting: we let $\mathscr{K}$ be a closed convex set in a real Banach space $\mathcal{E}$ and let $T: \mathscr{K} \rightarrow \mathcal{E}^{*}$. $T$ need not be

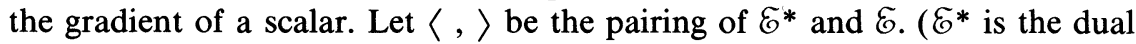
space of $\mathcal{E}_{\text {.) }}$ Corresponding to (5.5) we seek a $v \in \mathcal{K}$ that satisfies the variational inequality

$$
\langle T(v), w-v\rangle \geqslant 0 \quad \forall w \in \mathscr{K} .
$$

Even when $T$ is the gradient of a functional $\varphi$ defined on $\mathscr{K}$, there may be analytical advantages to seeking a $v$ satisfying (5.6) rather than minimizing $\varphi$.

Fichera (1964) analyzed Signorini's problem for linear elasticity by the direct methods of the calculus of variations, for which he refined some available lower semicontinuity theorems. He initiated the delicate regularity theory associated with determining the contact region and the reactions (Lagrange multipliers) supported there. Some of the abstract formalism for variational problems on convex sets had been developed earlier in optimal control theory, but this formalism did not deal with the regularity questions for partial differential equations of the sort that arise in Signorini's problem.

G. Duvaut reported to me that he had heard Fichera present his analysis of Signorini's problem at a conference in Bressanone, Italy in 1965 and that on his return to Paris, he had graduate students in solid mechanics study and expound Fichera's work. Thus Fichera's methods were publicized among mechanicians in the universities of Paris and throughout France. This dissemination of work on unilateral problems probably helped to establish the strong French activity in treating other unilateral problems (an activity in which Duvaut played a leading role. See the comments on applications below). 
At about the same time, Stampacchia $(1964,1965)$ confronted variational inequalities like (5.6) in his study of regularity of solutions of elliptic equations. (The characterization of a weak form of a sub-solution leads to an inequality of the form (5.6).) Over the next couple of years there was an intensive development of the theory of variational inequalities by Browder $(1965,1966)$, Hartman and Stampacchia (1966), Lions and Stampacchia (1967), Lewy (1968), Brezis (1968), Brezis and Stampacchia (1968), Lewy and Stampacchia (1969) and others. The abstract theory culminated in the marriage of variational inequalities with pseudomonotone operators in the hands of Brezis (1968). Cf. Lions (1969). Significant progress on the regularity of solutions was made in the works just listed. But many aspects of this question still remain to be resolved.

It is not clear to what extent Fichera's work influenced that of these authors, many of whom cited Fichera. (Cf. Fichera $(1972, \S 11)$.) At the least, the solution of Signorini's problem stood for several years as the main concrete application of the theory to classical physics. As such, it gave promise that the theory had significance beyond the confines of pure analysis.

This promise was soon realized. Numerous applications were made to a host of free surface problems in such diverse fields as plasticity, fluid dynamics, plasma physics, filtration, melting, etc. These are described, along with extensive bibliographies, in the texts of Duvaut and Lions (1972), Baiocchi and Capelo (1978), Kinderlehrer and Stampacchia (1980), and Friedman (1983). Indeed, the subject of variational inequalities now seems dominated by particular applications. The analysis of regularity of solutions, still the main source of difficulty, is forced to accommodate the peculiarities of each special class of problems.

An account of the many refinements in the treatment of Signorini's problem is given by Kinderlehrer (1981). Antman (1979, 1983) has used variational inequalities to handle (3.5) for ordinary differential equations of nonlinear elasticity by replacing it with a sequence of inequalities like $\operatorname{det}(\partial p / \partial x) \geqslant 1 / n$ and then obtaining sharp enough estimates to show that the resulting sequence of solutions converges to a solution whose Jacobian is positive. (Here again we find that a theory developed under the stimulus of one class of problems from elasticity has illuminated another class.)

6. Other contributions. John (1961) studied the rotation and strain in a deformable body with an eye toward justifying the validity of various plate theories by means of suitable estimates. For this purpose he used major new results on the Banach space BMO of functions of bounded mean oscillation developed by John and Nirenberg (1961) expressly for his problem and for the work of Moser (1961) on Harnack's inequality. (John and Nirenberg named BMO, which had appeared earlier in the study of conformal mappings.) BMO filled a gap in a natural scaling of function spaces (cf. Fefferman (1971) and Fefferman and Stein (1972)) and has since played a critical role in modern analysis.

Galerkin (1915) in studying elastic rods and plates introduced his projection method, generalizing that of Rayleigh and Ritz, which approximates solutions 
of differential equations by sequences of solutions of finite-dimensional problems. The convergence of the method was demonstrated by Keldysh (1942) (cf. Kantorovich and Krylov (1958) and Mikhlin (1966)). The method stimulated advances in numerical and functional analysis. Indeed, many developments in the theory of operators of monotone type (mentioned in \$4) and in the theory of variational inequalities rest on it.

The finite element methơd (cf. Oden (1972) and Ciarlet (1978)), introduced by Courant (1943), is a version of Galerkin's method, which is particularly effective for the numerical treatment of boundary value problems. Its usefulness was not appreciated at that time. The method in a different guise was rediscovered by Argyris (1954-1955) and by Turner, Clough, Martin and Topp (1956). It was thereafter intensively cultivated by engineers interested in structural mechanical problems of elasticity. (See Zienkiewicz (1973) for an historical account of this development.) That the finite element in the form used by engineers is but a specialization of Galerkin's method was soon recognized. In the 1960's mathematicians began a rigorous analysis of numerical errors for the method. According to Ciarlet (1976, pp. 106-107), on whose assessments I am relying in this paragraph, Zlámal (1968) gave the first general mathematical analysis of error in the finite element method. The continuing study of the method has spawned new developments in functional analysis, especially with respect to function spaces.

In his local analysis of buckling of elastic structures, Koiter (1945) gave a systematic treatment of imperfection methods. To appreciate what these are, we can write (2.9) in the even more compact form

$$
f(\lambda, u)=0 \quad \text { with } f(\lambda, 0)=0 .
$$

In imperfection studies, the function $f$ is embedded in a one-parameter family of functions with values $f(\lambda, \varepsilon, u)$ with $f(\lambda, 0, u)=f(\lambda, u)$ and $f(\lambda, \varepsilon, 0) \neq 0$ for $\varepsilon \neq 0$. In place of (6.1) we study the behavior of solutions of

$$
f(\lambda, \varepsilon, u)=0 \text {. }
$$

(An imperfection $\varepsilon$ can be introduced into (2.1), (2.4) by assuming that the initial shape of the rod is not straight. $\varepsilon$ could be taken as an amplitude of the initial curvature.) The dependence of $(\lambda, u)$ on $\varepsilon$ can give important insights into the stability of physical systems described by (6.2).

The local analysis of the generalization of (6.2) obtained by allowing $\varepsilon$ to be an $n$-tuple of real numbers has been carried out by Thom and Mather (cf. Golubitsky and Guillemin (1973), e.g.) by using methods of real algebraic geometry and resulted in catastrophe theory, or more generally, singularity theory. To the detriment of catastrophe theory, many of its first applications were directed to sociology rather than to the elaboration of the well-established path cut by Koiter. The methods of singularity theory have recently, however, succeeded in illuminating problems of elasticity. Moreover, the peculiarities of well-posed problems of elasticity have begun to influence the development of singularity theory. (Cf. Golubitsky and Schaeffer (1979), e.g.)

There are of course other areas in which the influence of elasticity has been felt. The theory of quasilinear hyperbolic systems has received much of its 
inspiration from gas dynamics. The nonlinear constitutive functions entering such systems for elastic media may have quite a form quite different from that for gases. An awareness of this fact is stimulating some new research directions in the basic theory. But it is premature to attempt an evaluation of the effect of elasticity.

7. Conclusion. The equations of nonlinear elasticity are parametrized by the tensor-valued function that gives the Piola-Kirchhoff stress as a function of the position gradient. This function differs from material to material. Thus in nonlinear elasticity one studies a whole class of materials, generating a whole class of nonlinear operators. This approach is in perfect accord with the philosophy of nonlinear operator theory.

Nevertheless, the abstract theories for nonlinear operators that have sprung up over the last twenty years have been largely incapable of treating nonlinear elasticity. Much of their generality was attained in directions irrelevant for elasticity. Those seeking to analyze the full equations of nonlinear elasticity had to develop their own operator theories, with inspiration from the modern abstract theories. As we have seen, this process has led to important developments in pure analysis.

The challenges offered by specific problems stimulated major advances. There was no technical obstacle that would have prevented Rabinowitz's Theorem from being proved, say in 1934, by Leray and Schauder. But Rabinowitz had the perspicacity to recognize the need for his theorem and the skill to surround it with a beautiful and useful theory.

A few years ago I was astounded to discover that some very eminent analysts were not aware that one could easily write down the exact equations for the large vibrations of an elastic string. This state of affairs was no doubt due to failure of virtually every elementary book on partial differential equations to produce an honest derivation of the wave equation. (The sole exception known to me is the text of Weinberger (1965).) By some mathematical version of Gresham's Law, the simple and convincing derivation of Euler (1771) was driven out of circulation and replaced by baser derivations, which are incompatible with the standards of precision demanded of modern analysis. $^{1}$

As the material of $\$ 3$ was meant to suggest, it is possible to present the theory of nonlinear elasticity (and indeed the theory of continuum mechanics) in a perfectly straightforward way, which proceeds inexorably from elementary geometry and from basic physical principles to well-set initial-boundary value problems. Nothing in the derivation need be incompatible with modern advanced calculus; the reader need not sacrifice his right to the clarity he would demand of mathematics. The role of nonlinear elasticity as an examplar of such a comprehensible mathematical science is not the least of its contributions to analysis.

\footnotetext{
${ }^{1}$ There is unfortunately a voluminous and growing literature devoted to doing poorly what Euler did well. Compared to the crimes of contemporary authors of such works, those of Greenhill were petty. It would help if this literature were confined to some newly founded journal, perhaps to be called Regressions in Applied Mathematics.
} 
ACKNOWLEDGEMENT. The research reported here was partially supported by NSF Grant MCS-80-01844. In preparing this work I benefitted from helpful discussions with M. G. Crandall, R. Di Perna, G. Duvaut, D. Kinderlehrer, F. Murat, U. Neri, P. H. Rabinowitz, L. Tartar, C. Truesdell, and J. H. Wolkowisky. Naturally, the interpretations I have given to the events discussed are solely my own.

\section{REFERENCES}

1. J. C. Alexander (1981), A primer on connectivity, Proc. Conf. on Fixed Point Theory, 1980 (E. Fadell and G. Fournier, eds.), Lecture Notes in Math., vol. 886, Springer-Verlag, Berlin and New York.

2. J. C. Alexander and S. S. Antman (1981), Global behavior of bifurcating multidimensional continua of solutions for multiparameter nonlinear eigenvalue problems, Arch. Rational Mech. Anal. 76, 339-354.

3. (1983), Global behavior of solutions of nonlinear equations depending on infinite-dimensional parameters, Indiana Univ. Math. J. 32.

4. J. C. Alexander and P. M. Fitzpatrick (1979), The homotopy of certain spaces of non-linear operators and its relation with global bifurcation of fixed points of parametrized condensing operators, J. Funct. Anal. 34, 87-106.

5. S. S. Antman (1978), Buckled states of nonlinearly elastic plates, Arch. Rational Mech. anal. 67, $111-149$.

6. (1979), The eversion of thick spherical shells, Arch. Rational Mech. Anal. 70, $113-123$.

7. (1983), Regular and singular problems for large elastic deformations of tubes, wedges, and cylinders, Arch. Rational Mech. Anal. (to appear).

8. S. S. Antman and C. S. Kenney (1981), Large buckled states of nonlinearly elastic rods under torsion, thrust, and gravity, Arch. Rational Mech. Anal. 76, 289-338.

9. S. S. Antman and G. Rosenfeld (1978), Global behavior of buckled states of nonlinearly elastic rods, SIAM Rev. 20, 513-566. Corrections and additions, ibid. 22 (1980), 186-187.

10. J. H. Argyris (1954-1955), Energy theorems and structural analysis, Part I: General theory, Aircraft Engineering 26, 347-356, 383-387, 394; 27, 42-58, 80-94, 125-134.

11. C. Baiocchi and A. Capelo (1978), Disequazioni variazionali e quasi variazionali. Applicazioni a problemi di frontiera libera, Two vols., Pitagora, Bologna.

12. J. M. Ball (1977a), Convexity conditions and existence theorems in nonlinear elasticity, Arch. Rational Mech. Anal. 63, 337-403.

13. (1977b), Constitutive inequalities and existence theorems in nonlinear elastostatics, in Nonlinear Analysis and Mechanics: Heriot-Watt Symposium, Vol. I, Pitman Research Notes in Math., no. 17 (R. Knops, ed.), Pitman, London, pp. 187-241.

14. (1980), Strict convexity, strong ellipticity, and regularity in the calculus of variations, Math. Proc. Cambridge Philos. Soc. 87, 501-513.

15. (1982), Discontinuous equilibrium solutions and cavitation in nonlinear elasticity, Philos. Trans. Roy. Soc. London Ser. A 306, 557-611.

16. __ ed. (1983), Systems of nonlinear partial differential equations, Reidel, Dordrecht.

17. M. S. Berger (1967a), On von Kármán's equations and the buckling of a thin elastic plate, I: The clamped plate, Comm. Pure Appl. Math. 20, 687-719.

18. (1967b), On nonlinear perturbations of the eigenvalues of a compact self-adjoint operator, Bull. Amer. Math. Soc. 73, 704-708.

19. (1969), On one parameter families of real solutions of nonlinear operator equations, Bull. Amer. Math. Soc. 75, 456-459.

20. M. S. Berger and P. Fife (1968), On von Kármán's equations and the buckling of a thin elastic plate. II, Comm. Pure Appl. Math. 21 (1968), 227-241.

21. R. Böhme (1971), Nichtlineare Störung der isolierte Eigenwerte sebstadjungierte Operatoren, Math. Z. 123, 61-92.

22. (1972), Die Lösung der Verzweigungsgleichungen für nichtlineare Eigenwertprobleme, Math. Z. 127, 105-126. 
23. H. Brezis (1968), Équations et inéquations non linéaires dans les espaces vectoriels en dualité, Ann. Inst. Fourier (Grenoble) 18, 115-175.

24. H. Brezis and G. Stampacchia (1968), Sur la régularité de la solution d'inéquations elliptiques, Bull. Soc. Math. France 96, 153-180.

25. F. E. Browder (1965), Nonlinear monotone operators and convex sets in Banach spaces, Bull. Amer. Math. Soc. 71, 780-785.

26. (1966a), On the unification of the calculus of variations and the theory of monotone non-linear operators in Banach space, Proc. Nat. Acad. Sci. U.S.A. 56, 419-425.

27. (1966b), Existence and approximation of solutions of non-linear variational inequalities, Proc. Nat. Acad. Sci. U.S.A. 56, 1080-1086.

28. P. G. Ciarlet (1978), The finite element method for elliptic problems, North-Holland, Amsterdam.

29. R. Courant (1943), Variational methods for the solution of problems of equilibrium and vibrations, Bull. Amer. Math. Soc. 49, 1-23.

30. M. G. Crandall and P. H. Rabinowitz (1970), Nonlinear Sturm-Liouville problems and topological degree, J. Math. Mech. 19, 1083-1102.

31. (1971), Bifurcation from simple eigenvalues, J. Funct. Anal. 8, 321-340.

32. B. Dacorogna (1982), Weak continuity and weak lower semicontinuity of non-linear functionals, Lecture Notes in Math., vol. 922, Springer-Verlag, Berlin and New York.

33. E. N. Dancer (1973), Global structure of the solutions of non-linear real analytic eigenvalue problems, Proc. London Math. Soc. (3) 27, 747-765.

34. R. DiPerna (1983), Convergence of approximate solutions to conservation laws, Arch. Rational Mech. Anal. 82, 27-70.

35. __ (1984), Convergence of the viscosity method for isentropic gas dynamics, Comm. Math. Phys. (to appear).

36. G. Duvaut and J. L. Lions (1972), Les inéquations en mecanique et en physique, Dunod, Paris, English transl., Springer-Verlag, Berlin and New York, 1976.

37. I. Ekeland and R. Temam (1972), Analyse convexe et problèmes variationnels, Dunod, Paris, English transl., North-Holland, Amsterdam, 1976.

38. J. L. Ericksen, Ill-posed problems in thermoelasticity theory, in [16].

39. L. Euler (1744), Additamentum I de curvis elasticis, methodus inveniendi lineas curvas maximi minimivi proprietate gaudentes, sive solutio problematis isoperimetrici latissimo sensu accepti, Bousquet, Lausanne and Geneva. in Opera Omnia, Ser. I, Vol. 24, Füssli, Zurich, 1960, pp. 231-297.

40. (1771), Genuina principia doctrinae de statu aequilibrii et motu corporum tam perfecte flexicilium quam elasticorum, Novi Comm. Acad. Sci. Petrop. 15 (1770), 381-413 in Opera Omnia, Ser. II, Vol. 11, Füssli, Zurich, pp. 37-61.

41. (1780), Determinatio onerum, quae columnae gestare valent, Acta. Acad. Sci. Petrop 2, 121-145; Examen insignis paradoxi in theoria columnarum occurentis, loc. cit., 146-162; De altitudine columnarum sub proprio pondere corruentium, loc. cit., 163-193. Reprinted in Opera Omnia, Ser. II, Vol. 17, Füssli, Zürich.

42. C. Fefferman (1971), Characterizations of bounded mean oscillation, Bull. Amer. Math. Soc. 77, 587-588.

43. C. Fefferman and E. M. Stein (1972), $H^{p}$ spaces of several variables, Acta Math. 129, $137-193$.

44. G. Fichera (1964), Problemi elastostatici con vincoli unilaterali: il problema di Signorini con ambigue condizioni al contorno, Mem. Accad. Naz. Lincei Ser. VII 7 (1964), 91-140; English transl. in Sem. Ist. Naz. di Alta Mat. 1962-63, Cremonese, pp. 613-679.

45. (1972), Boundary value problems of elasticity with unilateral constraints, Handbuch der Physik, Vol. VIa/2, Springer-Verlag, Berlin and New York, pp. 391-424.

46. P. M. Fitzpatrick, I. Massabò and J. Pejsachowicz (1983), Global several-parameter bifurcation and continuation theorems: a unified approach via complementing maps, Math. Ann. (to appear).

47. K. O. Friedrichs and J. J. Stoker (1941), The non-linear boundary value problem of the buckled plate, Amer. J. Math. 63, 839-888.

48. A. Friedman (1983), Variational principles and free-boundary problems, Wiley-Interscience, New York. 
49. B. G. Galerkin (1915), Rods and plates, Vestnik Inzh. 1 897-908. (Russian)

50. M. Giaquinta, The regularity problem of extremals of variational integrals, in [16].

51. E. Giusti, Some aspects of the regularity theory for nonlinear elliptic systems, in [16].

52. M. Golubitsky and V. Guillemin (1973), Stable mappings and their singularities, SpringerVerlag, Berlin and New York.

53. M. Golubitsky and D. Schaeffer (1979), A theory for imperfect bifurcation via singularity theory, Comm. Pure Appl. Math. 32, 21-98.

54. A. G. Greenhill (1881), Determination of the greatest height consistent with stability that a vertical pole or mast can be made, and of the greatest height to which a tree of given proportions can grow, Proc. Cambridge Philos. Soc. 4, 65-75.

55. (1883), On the strength of shafting when exposed both to torsion and to end thrust, Institution of Mechanical Engineers, Proc., pp. 182-209.

56. P. Hartman and G. Stampacchia (1966), On some non-linear differential functional equations, Acta Math. 115, 271-310.

57. J. Ize (1976), Bifurcation theory for Fredholm operators, Mem. Amer. Math. Soc. No. 174.

58. F. John (1961), Rotation and strain, Comm. Pure Appl. Math. 14, 391-413.

59. F. John and L. Nirenberg (1961), On functions of bounded mean oscillation, Comm. Pure Appl. Math. 14 (1961), 415-426.

60. L. V. Kantorovich and V. I. Krylov (1958), Approximate methods of higher analysis, English transl. of 3rd ed., Noordhoff, Groningen.

61. M. V. Keldysh (1942), On the method of B. G. Galerkin for the solution of boundary value problems, Izv. Akad. Nauk SSSR Ser. Mat. 6, 309-330.

62. H. B. Keller, J. B. Keller and E. L. Reiss (1962), Buckled states of circular plates, Quart. Appl. Math. 20, 55-65.

63. J. B. Keller and S. S. Antman, eds. (1969), Bifurcation theory and nonlinear eigenvalue problems, Benjamin, New York.

64. D. Kinderlehrer (1981), Remarks about Signorini's problem in linear elasticity, Ann. Scuola Norm. Sup. Pisa Cl. Sci. (4) 8, 605-645.

65. D. Kinderlehrer and G. Stampacchia (1980), Introduction to variational inequalities and their applications, Academic Press, New York.

66. W. T. Koiter (1945), Over de stabiliteit van het elastisch evenwicht, Thesis, Delft, English transl., NASA, Tech. Transl., F10, 833, 1967.

67. I. I. Kolodner (1955), Heavy rotating string-a non-linear eigenvalue problem, Comm. Pure Appl. Math. 8, 395-408.

68. M. A. Krasnosel'skii (1953), Application of variational methods to the problem of bifurcation points, Mat. Sb. 33. (Russian)

69. (1956), Topological methods in the theory of nonlinear integral equations, Gostekhteorizdat, English transl., Pergamon Press, New York, 1964.

70. H. Lewy (1968), On a variational problem with inequalities on the boundary, J. Math. Mech. 17, $861-884$.

71. H. Lewy and G. Stampacchia (1969), On the regularity of the solution of a variational inequality, Comm. Pure. Appl. Math. 22, 153-188.

72. J. L. Lions and G. Stampacchia (1967), Variational inequalities, Comm. Pure. Appl. Math. 20, $493-519$.

73. J. L. Lions (1969), Quelques méthodes de résolution des problèmes aux limites non linéaires, Dunod and Gauthier-Villars, Paris.

74. R. Magnus (1976), A generalization of multiplicity and the problem of bifurcation, Proc. London Math. Soc. (3) 32, 251-278.

75. J. B. McLeod and R. E. L. Turner (1976), Bifurcation for nondifferentiable operators with an application to elasticity, Arch. Rational Mech. Anal. 63, 1-45.

76. S. G. Mikhlin (1966), The numerical realization of variational methods, "Nauka", Moscow; English transl., The numerical performance of variational methods, Wolters-Noordhoff, Groningen, 1971.

77. C. B. Morrey, Jr. (1952), Quasi-convexity and the lower semicontinuity of multiple integrals, Pacific J. Math. 2, 25-53.

78. (1966), Multiple integrals in the calculus of variations, Springer-Verlag, Berlin and New York 
79. F. Murat (1978), Compacité par compensation, Ann. Scuola Norm. Sup. Pisa. Cl. Sci. 4 5, 489-507.

80.

(1981), Compacité par compensation: condition nécessaire et suffisante de continuité faible sous une hypothèse de rang constant, Ann. Scuola. Norm. Sup. Pisa Cl. Sci. 4 8, 69-102.

81. R. D. Nussbaum (1975), A global bifurcation theorem with applications to functional differential equations, J. Funct. Anal. 19, 319-338.

82. J. T. Oden (1972), Finite elements in nonlinear continua, McGraw-Hill, New York.

83. G. H. Pimbley, Jr. (1962), A sublinear Sturm Liouville problem, J. Math. Mech. 11, 121-138.

84. _ (1963), The eigenvalue problem for sublinear Hammerstein operators with oscillation kernels, J. Math. Mech. 12, 577-598.

85. P. H. Rabinowitz (1970), Nonlinear Sturm-Liouville problems for second order ordinary differential equations, Comm. Pure Appl. Math. 23, 939-961.

86. (1971), Some global results for nonlinear eigenvalue problems, J. Funct. Anal. 7, 487-513.

87. (1973), Some aspects of nonlinear eigenvalue problems, Rocky Mountain J. Math. 3, $161-202$.

88. E. L. Reiss (1965), Bifurcation buckling of spherical caps, Comm. Pure. Appl. Math. 18, $65-82$.

89. (1969), Column buckling - an elementary example of bifurcation, Bifurcation Theory and Nonlinear Eigenvalue Problems (J. B. Keller and S. S. Antman, eds.), Benjamin, New York, pp. $1-16$.

90. L. Saalschütz (1880), Der belastete Stab, Teubner, Leipzig.

91. A. Signorini (1959), Questioni di elasticità nonlinearizzata e semilinearizzata, Rend. Mat. 18, $1-45$.

92. G. Stampacchia (1964), Formes bilinéaires coercitives sur les ensembles convexes, C. R. Acad. Sci. Paris 258, 4413-4416.

93. (1965), Le problème de Dirichlet pour les équations elliptiques du second ordre à coefficients discontinus, Ann. Inst. Fourier (Grenoble) 15, 189-258.

94. C. A. Stuart (1973), Some bifurcation theory for $k$-set contractions, Proc. London Math. Soc. (3) 27, 531-550.

95. (1975), Spectral theory of rotating chains, Proc. Roy. Soc. Edinburgh Sect. A 73, $199-214$.

96. L. Tartar (1979), Compensated compactness and applications to partial differential equations, Nonlinear Analysis and Mechanics: Heriot-Watt Symposium, Vol. IV (R. Knops, ed.), Pitman, New York.

97. J. F. Toland (1976), Global bifurcation for $k$-set contractions without multiplicity assumptions, Quart. J. Math. Oxford Ser. (2) 27, 199-216.

98. V. A. Trenogin and V. I. Yudovich, eds. (1974), Bifurcation theory and nonlinear eigenvalue problems, Russian transl., “Mir”, Moscow, 1969. (See [63] for English original.)

99. C. Truesdell (1960), The rational mechanics of flexible or elastic bodies 1638-1788, L. Euleri Opera Omnia, Ser. II, Vol. $11_{2}$, Füssli, Zürich.

100. M. J. Turner, R. W. Clough, H. C. Martin and L. J. Topp (1956), Stiffness and deflection analysis of complex structures, J. Aero. Sci. 23, 805-823.

101. R. E. L. Turner (1970), Nonlinear eigenvalue problems with nonlocal operators, Comm. Pure. Appl. Math. 23, 963-972.

102. (1971), Transversality in nonlinear eigenvalue problems, in Contributions to Nonlinear Functional Analysis (E. Zarantonello, ed.), Academic Press, New York, pp. 37-68.

103. M. M. Vainberg (1956), Variational methods for the study of nonlinear operators, Gostekhteorizdat; English transl., Holden-Day, San Francisco, Calif., 1964.

104. I. I. Vorovich (1969), Some estimates of the number of solutions for the Kármán equations in connection with the problem of stability of plates and shells, Problems of Hydrodynamics and Mechanics of Continuous Media, for the sixtieth birthday of Academician L. I. Sedov, "Nauka", Moscow; pp. 762-771; English transl., SIAM, Philadelphia, Penn., 1969.

105. (1970), On the behavior of plates after loss of stability, Problems of Mechanics of Deformable Solid Bodies, for the sixtieth birthday of Academician V. V. Novozhilov, "Nauka", Moscow. 
106. (1955), On the behavior of circular plates after the loss of stability, Uchen. Zap. Rostov. Univ. 4, 55-60. (Russian)

107. (1958), Some questions on the stability of shells in the large, Dokl. Akad. Nauk SSSR 122, 37-40; English transl., Certain questions on the large deformation stability of shells, Soviet Math. Dokl. 3 (1958), 1032-1035.

108. H. F. Weinberger (1965), A first course in partial differential equations, Blaisdell, Waltham, Mass.

109. J. H. Wolkowisky (1967), Existence of buckled states of circular plates, Comm. Pure. Appl. Math. 20, 549-560.

110. (1969), Nonlinear Sturm-Liouville problems, Arch. Rational Mech. Anal. 35, 299-320.

111. O. C. Zienkiewicz (1973), Finite elements. The background story, in Mathematics of Finite Elements and Applications (J. R. Whiteman, ed.), Academic Press, New York, pp. 1-25.

112. M. Zlámal (1968), On the finite element method, Numer. Math. 12, 394-409.

113. J. Moser (1961), On Harnack's theorem for elliptic differential equations, Comm. Pure Appl. Math. 14, 577-591.

114. L. Tartar, The compensated compactness method applied to systems of conservation laws, in [16].

Department of Mathematics and Institute for Physical Science and Technology, University of Maryland, College Park, Maryland 20742 
